\title{
Surfaces on time scales and their metric properties
}

\author{
Sibel Paşalı Atmaca* and Ömer Akgüller
}

\author{
"Correspondence: sibela@mu.edu.tr \\ Department of Mathematics, \\ Faculty of Science, Mugla University, \\ Mugla, 48000, Turkey
}

\begin{abstract}
We present a theoretical framework for surfaces parameterized by the product of two arbitrary time scales. We also study surfaces by delta regular curves lying on them and give their metric tensor known as the first fundamental form with respect to partial delta derivatives.
\end{abstract}

Keywords: time scales; delta derivatives; vector fields; delta covariant; first fundamental form

\section{Introduction}

The theory of time scales, which has recently received a lot of attention, was introduced by Hilger [1] to unify continuous and discrete analysis. In the study [2], Bohner and Guseinov introduced the concepts such as curves, delta tangent lines, surfaces and delta tangent planes on time scales. Due to this significant paper, time scale calculus has attracted researchers of differential geometry [3-6]. In the paper [7], Bohner and Guseinov also gave a brief introduction to surfaces on time scales and discussed integrals on surfaces elaborately.

In this paper, we first study surfaces parameterized by the product of two arbitrary time scales. Since the parametrization lets us obtain a dynamic structure which involves discrete or continuous geometric data, we give the theoretical framework to surfaces in the viewpoint of manifolds. While studying, we only give the results with the delta differential operator, it is straightforward to obtain similar results for a backward differential operator. In [2], authors briefly introduced that partial delta tangential vectors help us to construct a tangent plane. We also deal with this idea by the surfaces which have less conditions like $\sigma_{i}$-complete differentiability. Throughout this paper, we use the chain rule that Bohner and Guseinov presented as follows.

Theorem 1 Let the function $f$ be $\sigma_{1}$-completely delta differentiable at the point $\left(t^{0}, s^{0}\right)$. If the functions $\varphi$ and $\psi$ have delta derivatives at $\xi^{0}$, then the composite function

$$
F(\xi)=f(\varphi(\xi), \psi(\xi)) \quad \text { for } \xi \in \mathbb{T}
$$

has a delta derivative at the point $\xi^{0}$, which is expressed by the formula

$$
F^{\Delta}\left(\xi^{0}\right)=\frac{\partial f\left(t^{0}, s^{0}\right)}{\Delta_{1} t} \varphi^{\Delta}\left(\xi^{0}\right)+\frac{\partial f\left(\sigma_{1}\left(t^{0}\right), s^{0}\right)}{\Delta_{2} s} \psi^{\Delta}\left(\xi^{0}\right)
$$

where $\varphi(\mathbb{T})=\mathbb{T}_{1}$ and $\psi(\mathbb{T})=\mathbb{T}_{2}$. 
Theorem 2 Let the function $f$ be $\sigma_{2}$-completely delta differentiable at the point $\left(t^{0}, s^{0}\right)$. If the functions $\varphi$ and $\psi$ have delta derivatives at $\xi^{0}$, then the composite function defined by (1) has a delta derivative at the point $\xi^{0}$, which is expressed by the formula

$$
F^{\Delta}\left(\xi^{0}\right)=\frac{\partial f\left(t^{0}, \sigma_{2}\left(s^{0}\right)\right)}{\Delta_{1} t} \varphi^{\Delta}\left(\xi^{0}\right)+\frac{\partial f\left(t^{0}, s^{0}\right)}{\Delta_{2} s} \psi^{\Delta}\left(\xi^{0}\right)
$$

where $\varphi(\mathbb{T})=\mathbb{T}_{1}$ and $\psi(\mathbb{T})=\mathbb{T}_{2}$.

Detailed proofs of chain rule theorems and significant remarks can be found in [2]. In [4], vector fields and covariant delta derivatives on time scales are studied for higher dimensional time scales. In Section 3, we present a delta covariant derivative by considering curves lying on the surface. We also study delta integral curves of a vector field which can be derived by a system of dynamic equations on a surface. Finally, in Section 4, we introduce a metric tensor which involves partial delta differentials. By using this tensor, we are able to calculate the length of a delta regular curve lying on the surface with the condition of increasing transformation.

We refer the reader to resources such as [8-11] for more detailed studies on the calculus of time scales and $[12,13]$ for details on the differential geometry of surfaces.

\section{Surfaces on time scales}

We may consider a surface $\mathcal{S}$ as a closed subset $\Lambda^{3}=\mathbb{T}_{1} \times \mathbb{T}_{2} \times \mathbb{T}_{3}$ of $\mathbb{R}^{3}$, where $\mathbb{T}_{i}, i=$ $1,2,3$, are arbitrary time scales. However, any closed subset of $\mathbb{R}^{3}$ may not be a surface. A theoretical study for any $\mathcal{S} \subset \mathbb{R}^{3}$ to be a surface is given in this section.

Definition 3 Let $\mathcal{S}$ be a closed subset of $\mathbb{R}^{3}$. $\mathcal{S}$ is a surface if for each point $P$ in $\mathcal{S}$, there is a neighborhood $A$ of $P$ and a function $\varphi: U \rightarrow \mathcal{S}$, where $U$ is a closed set in $\mathbb{R}^{2}$ and an open set in time scale topology, satisfying the following conditions:

i. $\varphi: U \rightarrow \mathbb{R}^{3}$ is $\Delta$-differentiable and for all $(t, s) \in U$

$$
\frac{\partial \varphi(t, s)}{\Delta_{1} t} \times \frac{\partial \varphi(t, s)}{\Delta_{2} s} \neq 0
$$

i.e., $\varphi$ is $\Delta$-regular.

ii. $\varphi(U)=\mathcal{S} \cap A$ and $\varphi: U \rightarrow \varphi(U)$ is a homeomorphism.

The function $\varphi: U \rightarrow \mathcal{S}$ is called a surface patch. $\mathcal{S}$ is called a smooth surface if for all points $P$ in $\mathcal{S}$, there exists a surface patch such that $P \in \varphi(U)$.

Speaking about time scale topology, we consider the opens as the sets whose closures are open in the standard real topology. We refer the readers who want to go further into the topic to [14]. Since $\varphi$ is $\Delta$-regular, one can also conclude that $\varphi$ belongs to the class $C_{\mathrm{rd}}^{\infty}$.

Proposition 4 Let $U \subset \mathbb{T}_{1} \times \mathbb{T}_{2}$ and let $f$ be a $\Delta$-differentiable function. Then the set

$$
\mathcal{S}=\left\{(t, s, f(t, s)) \mid(t, s) \in \mathbb{T}_{1} \times \mathbb{T}_{2}\right\}
$$


Proof Assume that the Euclidean coordinate system on a parameter set is $\{t, s\}$. Since coordinate functions $t$ and $s$ are $\Delta$-differentiable and $f$ is also $\Delta$-differentiable, we may conclude that $\varphi$ is $\Delta$-differentiable. The Jacobian matrix of $\varphi$ with respect to delta differentiation is

$$
J(\varphi)=\left(\begin{array}{cc}
\frac{\partial t}{\Delta_{1} t} & \frac{\partial t}{\Delta_{2} s} \\
\frac{\partial s}{\Delta_{1} t} & \frac{\partial s}{\Delta_{2} s} \\
\frac{\partial f(t, s)}{\Delta_{1} t} & \frac{\partial f(t, s)}{\Delta_{2} s}
\end{array}\right)=\left(\begin{array}{cc}
1 & 0 \\
0 & 1 \\
\frac{\partial f(t, s)}{\Delta_{1} t} & \frac{\partial f(t, s)}{\Delta_{2} s}
\end{array}\right),
$$

and since for all $P=\left(p_{1}, p_{2}\right) \in U, \operatorname{rank} J(\varphi)=2$, the function $\varphi$ is $\Delta$-regular. It is also trivial that $\varphi$ is homeomorphism.

Theorem 5 Let $U$ and $\tilde{U}$ be nonempty closed subsets of $\mathbb{R}^{2}$ and let $\varphi: U \rightarrow \mathcal{S}$ be a $\Delta$-regular surface patch. If $\phi: \tilde{U} \rightarrow U$ is diffeomorphism, then the function

$$
\tilde{\varphi}=\varphi \circ \phi: \tilde{U} \rightarrow \mathbb{R}^{3}
$$

is a $\Delta$-regular surface patch.

Proof For the function $\phi$, let $\phi(\tilde{t}, \tilde{s})=(t, s)$, where $(t, s) \in U$ and $(\tilde{t}, \tilde{s}) \in \tilde{U}$.

First, consider $\tilde{\varphi}$ is $\sigma_{1}$-completely $\Delta$-differentiable. By the chain rule, we obtain

$$
\frac{\partial \tilde{\varphi}}{\Delta_{(1)} \tilde{t}}=\frac{\partial t}{\Delta_{(1)} \tilde{t}} \frac{\partial \varphi}{\Delta_{1} t}+\frac{\partial s}{\Delta_{(1)} \tilde{t}} \frac{\partial \varphi^{\sigma_{1}}}{\Delta_{2} s}
$$

and

$$
\frac{\partial \tilde{\varphi}}{\Delta_{(2) \tilde{s}}}=\frac{\partial t}{\Delta_{(2) \tilde{s}}} \frac{\partial \varphi}{\Delta_{1} t}+\frac{\partial s}{\Delta_{(2)} \tilde{s}} \frac{\partial \varphi^{\sigma_{1}}}{\Delta_{2} s} .
$$

Thus

$$
\frac{\partial \tilde{\varphi}}{\Delta_{(1)} \tilde{t}} \times \frac{\partial \tilde{\varphi}}{\Delta_{(2)} \tilde{s}}=\left(\frac{\partial t}{\Delta_{(1)} \tilde{t}} \frac{\partial s}{\Delta_{(s)} \tilde{s}}-\frac{\partial t}{\Delta_{(s)} \tilde{s}} \frac{\partial s}{\Delta_{(1)} \tilde{t}}\right) \frac{\partial \varphi}{\Delta_{1} t} \times \frac{\partial \varphi^{\sigma_{1}}}{\Delta_{2} s} .
$$

The constant on the right-hand side of equation (2) is equal to the determinant of the Jacobian matrix

$$
J(\phi)=\left(\begin{array}{cc}
\frac{\partial t}{\Delta_{(1)} \tilde{t}} & \frac{\partial t}{\Delta_{(2)^{\tilde{s}}}} \\
\frac{\partial s}{\Delta_{(1)} \tilde{t}} & \frac{\partial s}{\Delta_{(2)^{\tilde{s}}}}
\end{array}\right) .
$$

Now, we need to consider the case that the function $\tilde{\varphi}$ is $\sigma_{2}$-completely $\Delta$-differentiable. If this holds, then we obtain

$$
\frac{\partial \tilde{\varphi}}{\Delta_{(1)} \tilde{t}} \times \frac{\partial \tilde{\varphi}}{\Delta_{(2) \tilde{s}}}=\left(\frac{\partial t}{\Delta_{(1)} \tilde{t}} \frac{\partial s}{\Delta_{(s) \tilde{s}}}-\frac{\partial t}{\Delta_{(s)} \tilde{s}} \frac{\partial s}{\Delta_{(1)} \tilde{t}}\right) \frac{\partial \varphi^{\sigma_{2}}}{\Delta_{1} t} \times \frac{\partial \varphi}{\Delta_{2} s} .
$$

The constant on the right-hand side of equation (3) is equal to the determinant of the Jacobian matrix $J(\phi)$. 


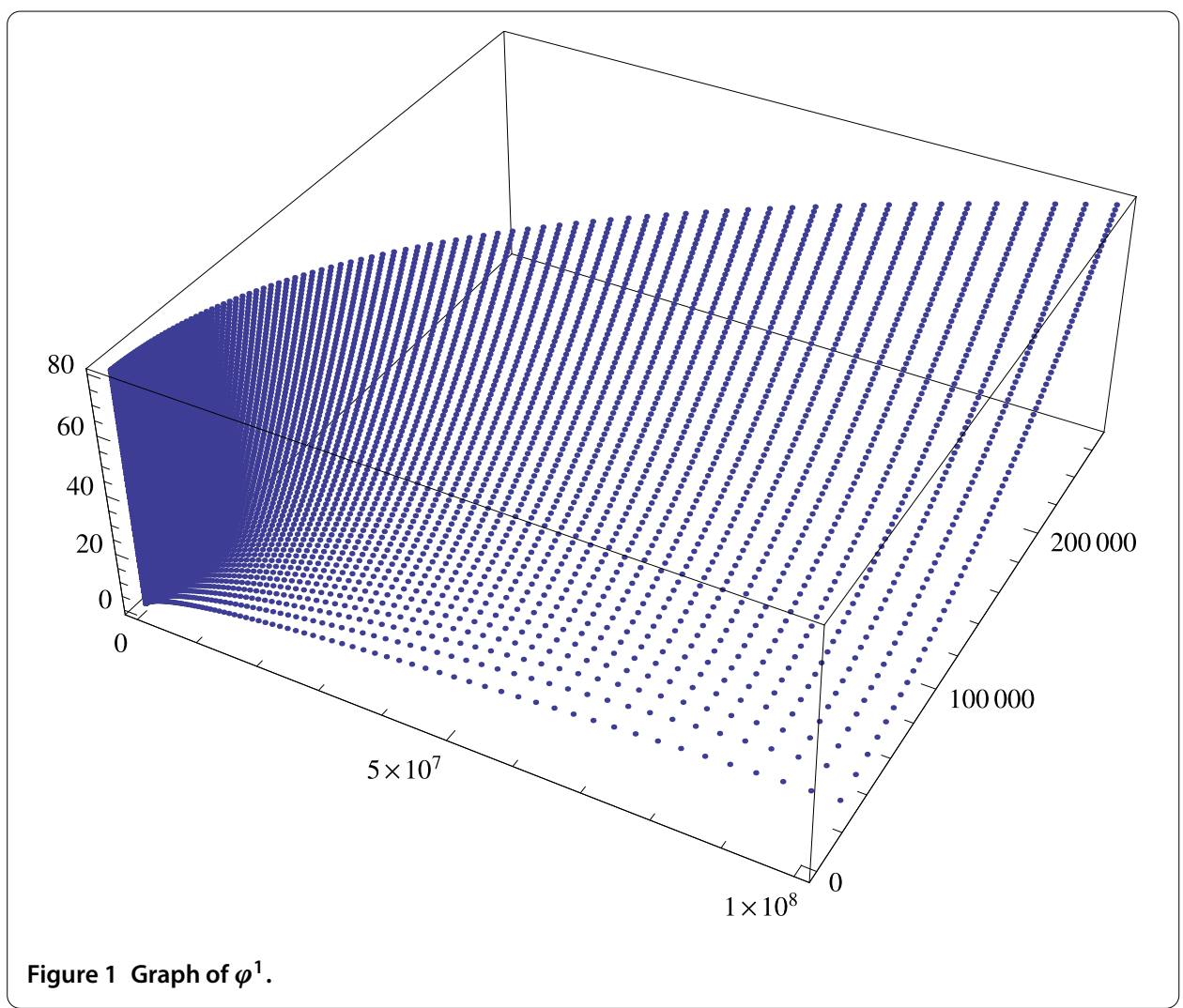

Since $\varphi$ is a $\Delta$-regular surface patch, one can say that Jacobian $J(\phi)$ never vanishes. Therefore, by equations (2) and (3), $\tilde{\varphi}$ is $\Delta$-regular.

Example 6 Let $\mathbb{T}_{1}=\left\{t^{2} \mid t \in \mathbb{Z}^{+}\right\}$and $\mathbb{T}_{2}=\left\{\sqrt{s} \mid s \in \mathbb{Z}^{+}\right\}$. Since $\varphi^{1}(t, s)=\left(t^{2}, 3 t s, s^{2}\right)$ is $\Delta$-regular, the image of $\varphi^{1}: \mathbb{T}_{1} \times \mathbb{T}_{2} \rightarrow \mathbb{R}^{3}$ defines a surface, and its graph will be as in Figure 1.

Example 7 Let $\mathbb{T}_{1}=\left\{\frac{1}{2} t \mid t \in \mathbb{Z}^{+}\right\}$and $\mathbb{T}_{2}=\left\{2^{s} \mid s \in \mathbb{N}_{0}\right\}$. It is easy to see $\varphi^{2}(t, s)=$ $\left(2 t, e_{1}(t, 0), e_{\frac{1-s}{s^{2}}}(s, 1)\right)$ is $\Delta$-regular. Therefore the image of $\varphi^{2}: \mathbb{T}_{1} \times \mathbb{T}_{2} \rightarrow \mathbb{R}^{3}$ defines a surface, and its graph will be as in Figure 2.

\subsection{Tangents and $\Delta$-derivatives}

The forward tangent line of a $\Delta$-regular curve $\Gamma$ on time scales is the straight line passing from the point $P$ of the curve through the point $P^{\sigma}$, and it has the vector $\Gamma^{\Delta}(P)$ as its direction vector [3]. The same idea can also be extended to the surfaces parameterized by time scales to obtain delta tangent planes [2].

A natural way to study a surface $\mathcal{S}$ is via the $\Delta$-regular curves $\Gamma$ that lie on $\mathcal{S}$.

Definition 8 A tangent vector to a surface $\mathcal{S}$ at a point $P \in \mathcal{S}$ is the tangent vector at $P$ of a curve in $\mathcal{S}$ passing through $P$. The set of all tangent vectors at $P$ is called a tangent space $T_{P}(\mathcal{S})$.

Theorem 9 Let $\varphi: U \rightarrow \mathcal{S}$ be a surface patch of $\mathcal{S}$ which contains $P \in \mathcal{S}$, let $(t, s)$ be the coordinates of $U$ and let $\Gamma$ be the $\Delta$-regular curve passing $P$. Then: 


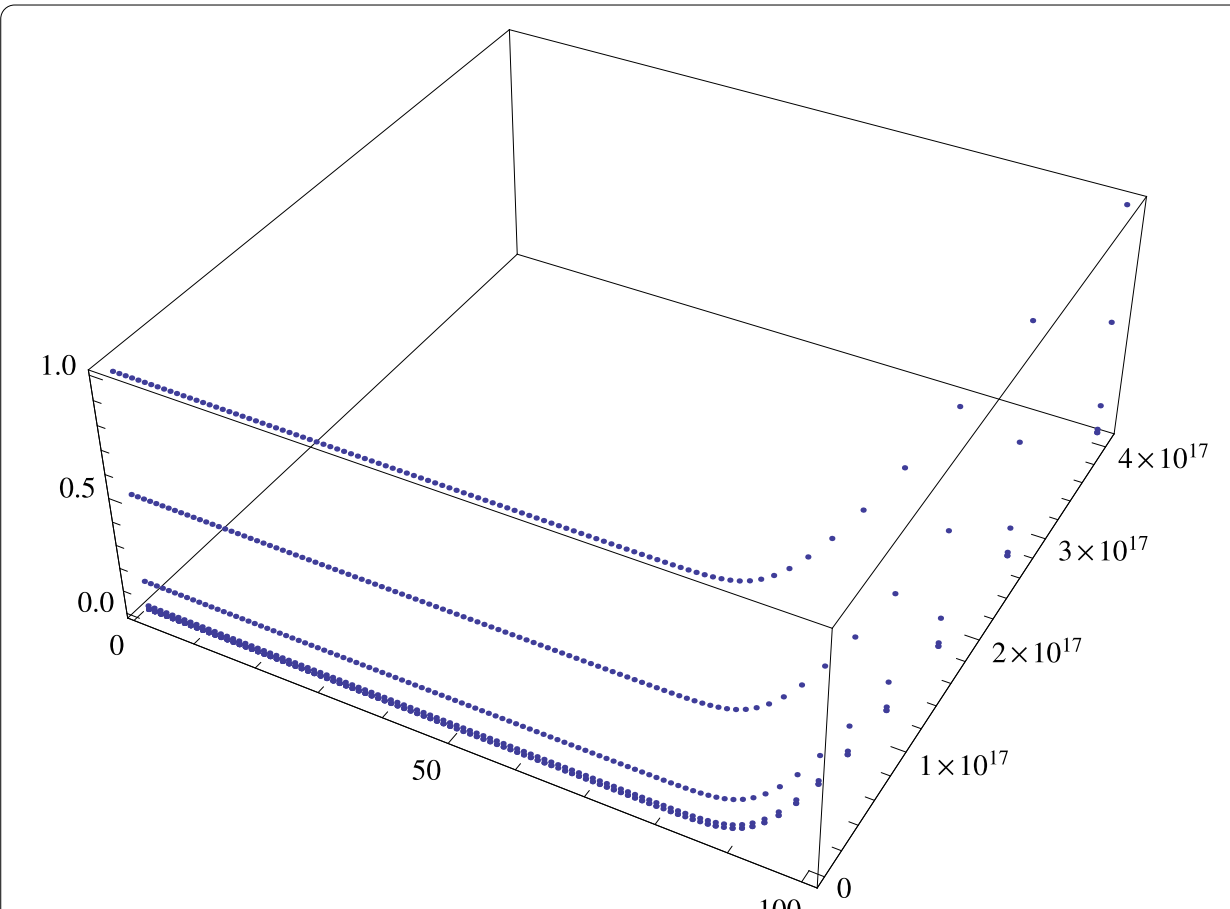

Figure 2 Graph of $\varphi^{2}$.

i. If $\Gamma$ is $\sigma_{1}$-completely $\Delta$-differentiable, then the tangent space at $P$ is spanned by the vectors $\frac{\partial \varphi\left(t_{0}, s_{0}\right)}{\Delta_{1} t}$ and $\frac{\partial \varphi\left(\sigma_{1}\left(t_{0}\right), s_{0}\right)}{\Delta_{2} s}$.

ii. If $\Gamma$ is $\sigma_{2}$-completely $\Delta$-differentiable, then the tangent space at $P$ is spanned by the vectors $\frac{\partial \varphi\left(t_{0}, \sigma_{2}\left(s_{0}\right)\right)}{\Delta_{1} t}$ and $\frac{\partial \varphi\left(t_{0}, s_{0}\right)}{\Delta_{2} s}$,

where $\varphi\left(t_{0}, s_{0}\right)=P$.

Proof Let $\Gamma$ be a $\Delta$-regular curve lying on $\mathcal{S}$ and $\Gamma(\xi)=\varphi(u(\xi), v(\xi))$ for $\xi \in \mathbb{T}$.

i. Suppose that $\Gamma$ is $\sigma_{1}$-completely $\Delta$-differentiable. Then, by the chain rule, we obtain

$$
\Gamma^{\Delta}=\frac{\partial \varphi}{\Delta_{1} t} t^{\Delta}+\frac{\partial \varphi^{\sigma_{1}}}{\Delta_{2} s} s^{\Delta} .
$$

Therefore, the tangent vector $\Gamma^{\Delta}$ is the linear combination of the vectors $\frac{\partial \varphi}{\Delta_{1} t}$ and $\frac{\partial \varphi^{\sigma_{1}}}{\Delta_{1} t}$.

Conversely, every vector on $\Lambda^{3} \subset \mathbb{R}^{3}$ can be written in the form of $\lambda \frac{\partial \varphi}{\Delta_{1} t}+\mu \frac{\partial \varphi^{\sigma_{1}}}{\Delta_{2} s}$ for such constants $\lambda$ and $\mu$.

Now, let us define

$$
\Gamma(x)=\varphi\left(t_{0}+\lambda x, s_{0}+\mu x\right) .
$$

For $x=0$, i.e., at $P \in \mathcal{S}$, the curve $\Gamma$ is a smooth curve. Therefore, we obtain

$$
\Gamma^{\Delta}=\lambda \frac{\partial \varphi}{\Delta_{1} t}+\mu \frac{\partial \varphi^{\sigma_{1}}}{\Delta_{2} s}
$$


This shows that every vector spanned by $\frac{\partial \varphi}{\Delta_{1} t}$ and $\frac{\partial \varphi^{\sigma_{1}}}{\Delta_{2} s}$ is a tangent vector of the curve $\Gamma$ on $\mathcal{S}$ at $P$.

ii. Similarly, assume that $\Gamma$ is $\sigma_{2}$-completely $\Delta$-differentiable. By the chain rule, we get

$$
\Gamma^{\Delta}=\frac{\partial \varphi^{\sigma_{2}}}{\Delta_{1} t} t^{\Delta}+\frac{\partial \varphi}{\Delta_{2} S} s^{\Delta} .
$$

Therefore, the tangent vector $\Gamma^{\Delta}$ is the linear combination of vectors $\frac{\partial \varphi^{\sigma_{2}}}{\Delta_{1} t}$ and $\frac{\partial \varphi}{\Delta_{1} t}$.

It is also possible to find such constants $\lambda^{\prime}$ and $\mu^{\prime}$, where every vector on $\Lambda^{3} \subset \mathbb{R}^{3}$ is in the form of $\lambda^{\prime} \frac{\partial \varphi^{\sigma_{2}}}{\Delta_{1} t}+\mu^{\prime} \frac{\partial \varphi}{\Delta_{2} s}$.

If we define a smooth curve

$$
\Gamma(x)=\varphi\left(t_{0}+\lambda^{\prime} x, s_{0}+\mu^{\prime} x\right)
$$

then we may obtain

$$
\Gamma^{\Delta}=\lambda^{\prime} \frac{\partial \varphi^{\sigma_{2}}}{\Delta_{1} t}+\mu^{\prime} \frac{\partial \varphi}{\Delta_{2} S}
$$

This shows that every vector spanned by $\frac{\partial \varphi^{\sigma_{2}}}{\Delta_{1} t}$ and $\frac{\partial \varphi}{\Delta_{2} s}$ is a tangent vector of the curve $\Gamma$ on $\mathcal{S}$ at $P$.

\section{Vector fields and covariant $\boldsymbol{\Delta}$-derivative}

Let $\sigma_{i}$ and $\Delta_{i}$ denote, respectively, a forward jump operator and a delta operator of $\mathbb{T}_{i}$ for $i \in\{1,2, \ldots, n\}, n \in \mathbb{N}$, where $\mathbb{T}_{i}$ are arbitrary time scales. Let us set

$$
\Lambda^{n}=\mathbb{T}_{1} \times \mathbb{T}_{1} \times \cdots \times \mathbb{T}_{n} .
$$

$\Lambda^{n}$ is called an $n$-dimensional time scale.

A vector field $X$ on $\Lambda^{n}$ is a function that assigns to each point $P \in \Lambda^{n}$ a tangent vector $v_{P}$.

Definition 10 Let $X$ be a vector field and $X(P) \in T_{P}\left(\Lambda^{n}\right)$. Generally, a vector field is denoted by

$$
X=\sum_{i=1}^{n} \alpha_{i} \frac{\partial}{\Delta_{i} x_{i}}
$$

where $\alpha_{i}$ are Euclidean coordinate functions and the set $\left\{\partial / \Delta_{1} x_{1}, \ldots, \partial / \Delta_{n} x_{n}\right\}$ is the natural basis for $T_{P}\left(\Lambda^{n}\right)$.

Relationship between a vector field $X$ and its Euclidean coordinate function $\alpha_{i}$ can be considered as follows: if each $\alpha_{i}$ of $X$ is $\sigma_{j}$-completely $\Delta$-differentiable, then one can say that $X$ is $\sigma_{j}$-completely $\Delta$-differentiable.

Suppose that $X$ is a vector field on $\Lambda^{n}$ and $v_{P} \in T_{P}\left(\Lambda^{n}\right)$. Consider the vector field $X \circ \beta$, where $\beta: \mathbb{T} \rightarrow \Lambda^{n}$ is defined by $\beta(t)=P+t v$. It is obvious to see that $X \circ \beta$ is a vector field on $\beta$. Also, $\beta(\mathbb{T})$ is the closed line parallel to the vector $v$ and $(X \circ \beta)^{\Delta} \in T_{P}\left(\Lambda^{n}\right)$.

Definition 11 The vector $(X \circ \beta)^{\Delta}(0)$ is called a covariant $\Delta$-derivative of $X$ in the direction of $v_{P}$ and denoted by $D_{v_{P}}^{\Delta} X$. 
Now we shall consider $\Delta$-integral curves of vector fields on surfaces. Let $\mathcal{S}$ be a smooth surface and let $X$ be a vector field on $\mathcal{S}$. A curve $\Gamma:[a, b] \rightarrow \mathcal{S}$, where $[a, b]$ is a nonempty closed set of $\mathbb{R}$, on $\mathcal{S}$ is called a $\Delta$-integral curve of $X$ if the vector $\Gamma^{\Delta} \in T_{\Gamma(t)} \mathcal{S}(t \in[a, b])$ at each point coincides with the value of $X$ at that point.

It is possible to find equations that should be satisfied by the $\Delta$-integral curve through an arbitrary point $P \in \mathcal{S}$. Let $\left\{x_{1}, x_{2}, x_{3}\right\}$ be a local coordinate system around $P$. Then $X$ is locally expressed as

$$
X=\sum_{i=1}^{3} \alpha_{i}(x) \frac{\partial}{\Delta_{i} x_{i}} .
$$

Let $\Gamma:[a, b] \rightarrow \mathcal{S}$ be the required $\Delta$-integral curve, and, for the sake of simplicity, choose a parameter such that $\Gamma(0)=P$. If we describe the position of $\Gamma(t)$ by the local coordinate system as $\Gamma(t)=\left(x_{1}(t), x_{2}(t), x_{3}(t)\right)$, then we obtain

$$
\Gamma^{\Delta}(t)=\sum_{i=1}^{3} \frac{d x_{i}}{\Delta t} \frac{\partial}{\Delta_{i} x_{i}} .
$$

Thus, the required equation is

$$
\frac{d x_{i}}{\Delta t}=\alpha_{i}\left(x_{1}(t), x_{2}(t), x_{3}(t)\right) \quad(i=1,2,3) .
$$

The condition of passing through the point $P$ at $t=0$ is expressed as the initial condition $x_{i}(0)=x_{i}(P)$.

By the way, (4) is a system of ordinary linear dynamic equations of first order. Theorem 5.8 in [8] assures us that this system has a unique solution if a coefficient matrix of the system is regressive.

By the existence of the solution, we see that there exists a $\Delta$-integral curve through an arbitrary point $P$ when $t=0$. We shall consider extending the domain of the integral curve as long as possible to assure the maximality. Since the solution of the system is unique, if two $\Delta$-integral curves pass through the same point at the same time, then they are connected as a single integral curve. So, the way of extending is unique. From this point of view, for each $P \in \mathcal{S}$, there exists a $\Delta$-integral curve $\Gamma(P)$ through the point when $t=0$, and it cannot be extended any more. This kind of $\Delta$-integral curves are called maximal, and we can conclude that $\mathcal{S}$ is covered by all the maximal $\Delta$-integral curves which are pairwise disjoint.

\section{Metric properties of surfaces on time scales}

The metric of a surface $\mathcal{S}$ is determined by the partial $\Delta$-derivatives of the surface patch $\varphi$. Assuming that $\varphi_{t}^{\Delta_{1}} \times \varphi_{s}^{\Delta_{2}} \neq 0$, the tangent plane to $\mathcal{S}$ is spanned by the two tangent vectors $\varphi_{t}^{\Delta_{1}}$ and $\varphi_{s}^{\Delta_{2}}$. The surface normal vector is orthogonal to both tangent vectors and can be computed as

$$
\vec{n}=\frac{\varphi_{t}^{\Delta_{1}} \times \varphi_{s}^{\Delta_{2}}}{\left\|\varphi_{t}^{\Delta_{1}} \times \varphi_{s}^{\Delta_{2}}\right\|} .
$$




\subsection{First fundamental form}

The Jacobian matrix encodes the metric of the surface in a way that it allows measuring transformation of angles, distances and areas by the mapping from the parameter domain to the surface.

Let $\omega_{1}$ and $\omega_{2}$ be two unit vectors in a parameter space. Cosine of the angle can be computed by the Euclidean dot product $\omega_{1}^{T} \omega_{2}$. When we consider the dot product of tangent vectors on $\mathcal{S}$, we obtain

$$
\widetilde{\omega}_{1}^{T} \widetilde{\omega_{2}}=\left(J \omega_{1}\right)^{T}\left(J \omega_{2}\right)=\omega_{1}^{T}\left(J^{T} J\right) \omega_{2}
$$

Therefore, the matrix product $J^{T} J$ induces the metric on a surface on time scales.

Definition 12 Let $\mathcal{S}$ be a surface on time scales. The first fundamental form of $\mathcal{S}$ is the assignment to each $P \in \mathcal{S}$ of the inner product,

$$
\cdot: T_{P}(\mathcal{S}) \times T_{P}(\mathcal{S}) \rightarrow \mathbb{R}
$$

i.e., the restriction of the Euclidean dot product to the $\Delta$-tangent space of $\mathcal{S}$, and can be shown by I.

Given a surface patch $\varphi: U \rightarrow \mathcal{S}$, the matrix for the first fundamental form with respect to the basis $\left\{\varphi_{t}^{\Delta_{1}}, \varphi_{s}^{\Delta_{2}}\right\}$ is

$$
\mathrm{I}=\left(\begin{array}{ll}
E & F \\
F & G
\end{array}\right)
$$

where $E=\varphi_{t}^{\Delta_{1}} \varphi_{t}^{\Delta_{1}}, F=\varphi_{t}^{\Delta_{1}} \varphi_{s}^{\Delta_{2}}$ and $G=\varphi_{s}^{\Delta_{2}} \varphi_{s}^{\Delta_{2}}$.

\subsection{Length measurement}

Since the first fundamental form I defines a metric on a surface, we can measure the length of a $\Delta$-regular curve $\varphi(x)=\varphi(\Gamma(x))$, defined as the image of a $\Delta$-regular curve $\Gamma(x)=$ $(t(x), s(x))$ in the parameter domain, where $x \in \widetilde{\mathbb{T}}$ for an arbitrary time scale.

Definition 13 Let $\mathbb{T}$ be a time scale. The function $\Gamma$ is called a path on $\mathbb{T}_{1} \times \mathbb{T}_{2}$ if it is increasing. Moreover, the composite function $\varphi \circ \Gamma: \mathbb{T} \rightarrow \mathcal{S}$ is called a path on the surface, where $\varphi$ is a $\Delta$-regular surface patch.

Theorem 14 Let $\Gamma:[a, b] \rightarrow \mathcal{S}$ be a path on the surface $\mathcal{S}$ and let $\varphi: U \rightarrow \mathcal{S}$ be a proper patch in $\mathcal{S}$, and suppose that $\Gamma$ is contained in this patch.

For the coordinate functions $t^{\prime}: \widetilde{\mathbb{T}} \rightarrow \mathbb{R}$ and $s^{\prime}: \widetilde{\mathbb{T}} \rightarrow \mathbb{R}$ of the path $\Gamma$, the length of $\Gamma$ is

$$
l(\Gamma)=\int_{a}^{b} \sqrt{E\left(\frac{d t^{\prime}}{\widetilde{\Delta} x}\right)^{2}+2 F\left(\frac{d t^{\prime}}{\widetilde{\Delta} x} \frac{d s^{\prime}}{\widetilde{\Delta} x}\right)+G\left(\frac{d s^{\prime}}{\widetilde{\Delta} x}\right)^{2}} \widetilde{\Delta} x,
$$

where $x \in[a, b] \subset \widetilde{\mathbb{T}}$ and $\widetilde{\Delta}$ is the corresponding delta-differential operator.

Proof Let $t^{\prime}(\widetilde{\mathbb{T}}):=\mathbb{T}_{1}^{\prime}$ and $s^{\prime}(\widetilde{\mathbb{T}}):=\mathbb{T}_{2}^{\prime}$, and let their $\Delta$-differential operators be $\Delta_{1}^{\prime}$ and $\Delta_{2}^{\prime}$, respectively. By considering the coordinate changing idea presented in Theorem 5 , we can 
express $\Gamma$ in terms of coordinates $\widetilde{\Gamma}=\varphi^{-1} \circ \Gamma:[a, b] \subset \widetilde{\mathbb{T}} \rightarrow U, \widetilde{\Gamma}(x)=\left(t^{\prime}(x), s^{\prime}(x)\right)$. Then $\Gamma(x)=\varphi(\widetilde{\Gamma}(x))$, hence $\Gamma(x)=\varphi\left(t^{\prime}(x), s^{\prime}(x)\right)$. By the chain rule, we can obtain

$$
\frac{d \varphi}{\widetilde{\Delta} x}=\frac{d t^{\prime}}{\widetilde{\Delta} x} \frac{\partial \varphi}{\Delta_{1}^{\prime} t^{\prime}}+\frac{d s^{\prime}}{\widetilde{\Delta} x} \frac{\partial \varphi}{\Delta_{2}^{\prime} s^{\prime}}=\sum_{i} \frac{d \varphi_{i}}{\widetilde{\Delta} x} \frac{\partial \varphi}{\Delta_{i}^{\prime} \varphi_{i}},
$$

where $\varphi_{i}$ is the $i$ th component of $\varphi$. This shows that $d t^{\prime} / \widetilde{\Delta} x$ and $d s^{\prime} / \widetilde{\Delta} x$ are the components of the $\Delta$-tangential vector with respect to basis $\left\{\partial \varphi / \Delta_{1}^{\prime} t^{\prime}, \partial \varphi / \Delta_{2}^{\prime} s^{\prime}\right\}$.

Now, speed in surface coordinates can be computed as

$$
\begin{aligned}
\left|\frac{d \varphi}{\widetilde{\Delta} x}\right| & =\sqrt{\left\langle\frac{d \varphi}{\widetilde{\Delta} x}, \frac{d \varphi}{\widetilde{\Delta} x}\right\rangle} \\
& =\sqrt{\left\langle\sum_{i}^{2} \frac{d \varphi_{i}}{\widetilde{\Delta} x} \frac{\partial \varphi}{\Delta_{i}^{\prime} \varphi_{i}}, \frac{d \varphi_{i}}{\widetilde{\Delta} x} \frac{\partial \varphi}{\Delta_{i}^{\prime} \varphi_{i}}\right\rangle} \\
& =\sqrt{\sum_{i, j}^{2} \frac{d \varphi_{i}}{\widetilde{\Delta} x} \frac{d \varphi_{j}}{\widetilde{\Delta} x}\left\langle\frac{\partial \varphi}{\Delta_{i}^{\prime} \varphi_{i}}, \frac{\partial \varphi}{\Delta_{j}^{\prime} \varphi_{j}}\right\rangle} .
\end{aligned}
$$

For length, we have

$$
\begin{aligned}
& l(\Gamma)=\int_{a}^{b} \sqrt{\frac{\partial \varphi}{\Delta_{1}^{\prime} t^{\prime}}\left(\frac{d t^{\prime}}{\widetilde{\Delta} x}\right)^{2}+2 \frac{\partial \varphi}{\Delta_{1}^{\prime} t^{\prime}} \frac{\partial \varphi}{\Delta_{2}^{\prime} s^{\prime}}\left(\frac{d t^{\prime}}{\widetilde{\Delta} x} \frac{d s^{\prime}}{\widetilde{\Delta} x}\right)+\frac{\partial \varphi}{\Delta_{2}^{\prime} s^{\prime}}\left(\frac{d s^{\prime}}{\widetilde{\Delta} x}\right)^{2}} \widetilde{\Delta} x \\
& =\int_{a}^{b} \sqrt{E\left(\frac{d t^{\prime}}{\widetilde{\Delta} x}\right)^{2}+2 F\left(\frac{d t^{\prime}}{\widetilde{\Delta} x} \frac{d s^{\prime}}{\widetilde{\Delta} x}\right)+G\left(\frac{d s^{\prime}}{\widetilde{\Delta} x}\right)^{2}} \widetilde{\Delta} x \text {. }
\end{aligned}
$$

\section{Competing interests}

The authors declare that they have no competing interests.

\section{Authors' contributions}

Authors contributed equally in writing this article. Authors read and approved the final manuscript.

\section{Acknowledgements}

The authors would like to thank the editor and the referees for their useful comments and remarks.

Received: 15 November 2012 Accepted: 28 May 2013 Published: 17 June 2013

\section{References}

1. Hilger, S: Analysis on measure chains: a unified approach to continuous and discrete calculus. Results Math. 18(1-2), 18-56 (1990)

2. Bohner, M, Guseinov, GS: Partial differentiation on time scales. Dyn. Syst. Appl. 13(3-4), 351-379 (2004)

3. Guseinov, GS, Ozyilmaz, E: Tangent lines of generalized regular curves parametrized by time scales. Turk. J. Math. 25(4), 553-562 (2001)

4. Ozyilmaz, E: Directional derivative of vector field and regular curves on time scales. Appl. Math. Mech. 27(10), 1349-1360 (2006)

5. Atmaca, SP: Normal and osculating planes of $\Delta$-regular curves. Abstr. Appl. Anal. 2010, Article ID 923916 (2010). doi:10.1155/2010/923916

6. Kusak, H, Caliskan, A: The delta nature connection on time scale. J. Math. Anal. Appl. 375, $323-330$ (2011)

7. Bohner, M, Guseinov, GS: Surface areas and surface integrals on time scales. Dyn. Syst. Appl. 19(3-4), 435-453 (2010)

8. Bohner, M, Peterson, A: Dynamic Equations on Time Scales: An Introduction with Applications. Birkhäuser, Boston (2001)

9. Bohner, M, Peterson, A: Advances in Dynamic Equations on Time Scales. Birkhäuser, Boston (2003)

10. Atici, FM, Guseinov, GS: On Green's functions and positive solutions for boundary value problems on time scales. J. Comput. Appl. Math. 141(1-2), 75-99 (2002)

11. Bohner, M, Guseinov, GS: Line integrals and Green's formula on time scales. J. Math. Anal. Appl. 326(2), 1124-1141 (2007) 
12. Kreyszig, E: Differential Geometry. Dover, New York (1991)

13. Do Carmo, MP: Differential Geometry of Curves and Surfaces. Prentice Hall, Englewood Cliffs (1976)

14. Oberste-Vorth, RW: The fell topology for dynamic equations on time scales. Nonlinear Dyn. Syst. Theory 9(4), 407-414 (2009)

doi:10.1186/1687-1847-2013-170

Cite this article as: Atmaca and Akgüller: Surfaces on time scales and their metric properties. Advances in Difference Equations 2013 2013:170.

Submit your manuscript to a SpringerOpen ${ }^{\circ}$ journal and benefit from:

- Convenient online submission

- Rigorous peer review

- Immediate publication on acceptance

- Open access: articles freely available online

- High visibility within the field

- Retaining the copyright to your article

Submit your next manuscript at $>$ springeropen.com 